\title{
ITINERARIO DE ESTUDIOS VIQUIANOS
}

\author{
Maurizio Martirano \\ (Universidad de Potenza, Basilicata)
}

RESUMEN: El trabajo da cuenta, breve y sintéticamente, de algunos estudios del Autor sobre la difusión del pensamiento de Vico en la cultura filosófica y política italiana de principios del siglo XIX.

Palabras Clave: Vico, $350^{\circ}$ Aniversario, viquismo, filosofía política, G. Ferrari, C. Cattaneo, M. Martirano.

\section{Itinerary of Vichian studies}

ABSTRACT: This work gives a brief account of some of the author's studies on the diffusion of Vico's thought in early Nineteenth Century Italian philosophical and political culture.

KeYwords: Vico, $350^{\text {th }}$ Anniversary, viquism, political philosophy, G. Ferrari, C. Cattaneo, M. Martirano.

\section{Itinerario di studi vichiani}

RIASSUNTO: Il contributo offre un resoconto breve e sintetico di alcuni studi dell'Autore sulla diffusione del pensiero di Vico nella cultura filosofica e politica italiana del primo Ottocento.

Parole Chiave: Vico, $350^{\circ}$ Anniversario, vichismo, filosofia politica, G. Ferrari, C. Cattaneo, M. Martirano.

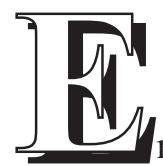

ntre los lugares más notables que señalan de manera emblemática la presencia de Vico en Nápoles, es fácil indicar el itinerario que conecta los talleres de San Biagio dei Librai con el Palacio Filomarino, la Biblioteca de los Girolamini y la Basílica del Gesù Vecchio, situada junto al Cortile delle Statue. Allí, después del desastroso terremoto de noviembre de 1980 (que habría abierto un difícil período para la ciudad, transformándole radicalmente el rostro), tuvieron lugar las lecciones universitarias durante algunos años. Y propiamente entre las aulas de la adyacente Accademia Pontaniana y las que se asoman sobre el Cortile -donde son conservadas las efigies de autorizados representantes de una cultura napolitana impregnada de elementos europeos, algunos de los cuales han realizado una contribución fun- 
damental a la difusión del pensamiento de Vico- he tenido la oportunidad de asistir a mi primer curso universitario dedicado a la Scienza nuova, ofrecido por Fulvio Tessitore poco después de la muerte de Pietro Piovani, ${ }^{1}$ el cual, como es sabido, ha dado inicio a una nueva y rica época de investigaciones sobre el filósofo napolitano, que han encontrado una forma original tanto en los estudios como en la organización de la actividad del entonces denominado "Centro di studi vichiani", órgano del CNR. Precisamente, en el "Centro" he iniciado mis primeras investigaciones sobre la fortuna del pensamiento viquiano, orientadas a la reconstrucción de algunas tramas de la historia de la cultura y de la política italianas de los siglos dieciocho y diecinueve. Se trata de una época en la cual el filósofo de la Scienza nuova ha sido un tópos imprescindible, utilizado tanto por la cultura católica moderada como por la liberal. Dejando a un lado la cuestión de la fortuna o la desgracia italiana del «Vico viviente» (como ha definido Benedetto Croce en su Bibliografia vichiana), ante una extensión tan amplia ejercitada esa vez solo exteriormente, mis estudios, sin ninguna intención "vicocéntrica", se concentraron sobre dos aspectos en particular: por un lado, la influencia ejercida respecto de los reformistas napolitanos de la segunda mitad del siglo XVIII, en particular Grimaldi, Genovesi, Pagano y Delfico, estudiosos que han tenido un rol decisivo para la penetración y la difusión de las ideas viquianas entre los exiliados de la revolución del '99; por el otro, la acción que precisamente los exiliados napolitanos han ejercido sobre la cultura meridional, gracias a una operación de "restauración crítica del viquismo", a través de un complejo entramado que involucra también a otros autores y áreas geográficas. En efecto, se puede afirmar que nace el sentido más auténtico de un "viquismo" cuando Vincenzo Cuoco, Francesco Lomonaco y Francesco Saverio Salfi, partiendo de una común inspiración genovesiana, han asumido una actitud original frente a la reflexión del napolitano, utilizándola junto con la filosofía iluminista y la ideología, declinadas en sentido humanológico, vale decir en la dirección de una ciencia del hombre que rechaza cualquier tipo de abstracción metafísica, para dirigirse a los aspectos éticos y civiles. Una polivalencia de voces que se concentra en torno a la idea de leer la filosofía viquiana como investigación de una ciencia racional de los principios, de una indagación capaz de determinar el significado de los hechos históricos en una dimensión civil de la política, en cuanto lugar de constitución de la legislación, por la cual ella se muestra cercana a la tradición reformista e iluminista signada por la exigencia de conjugar los elementos teóricos con su necesaria verificación filológica-historiográfica. La indagación en torno a la línea de continuidad que va de Vico a Genovesi, hasta los exiliados de la revolución

1. No siendo posible desarrollar el discurso, sin embargo, es necesario, al menos, mencionar el hecho de que, privilegiando una metodología kulturgeschichtlich, Piovani ha hecho una contribución fundamental a la interpretación y a la difusión de una imagen del filósofo napolitano ya no como aislado y extraño, aunque sea en calidad de "precursor" a su tiempo, sino plenamente colocado dentro de un determinado contexto histórico cultural. 
napolitana permite, entonces, poner en evidencia los temas de la filosofía de la historia y de la política como clave para una lectura moderna de la auctoritas a lo largo de un recorrido que, desde el análisis histórico de los acontecimientos y los conflictos humanos, conduce a una visión del derecho y del Estado como necesidad de obligación política y de codificación racional. Encontrándose en el origen de tal proceso de modernización y de laicización de la experiencia práctica del actuar humano, Vico puede ser interpretado como un crítico de la tradición, pero, sobre todo, es con Vico y a través de Vico que se ha podido mirar el pasado para identificar las raíces históricas de los males de la sociedad meridional. Son problemáticas evidentes sobre todo en las reflexiones de Vincenzo Cuoco, autor en el cual el encuentro entre viquismo e ideología ha actuado de manera particular, contribuyendo a fecundar algunos motivos de la cultura lombarda de comienzos del Siglo Diecinueve. De hecho, la idea de privilegiar la dimensión histórica, la crítica a la excesiva curvatura de los hechos sobre premisas especulativas y a la "excesiva metafísica" del sistema viquiano, tanto como el énfasis de algunos elementos que han actuado extensamente en la historia del viquismo italiano -como, por ejemplo, el aislamiento sustancial del filósofo en su tiempo y su capacidad de anticipar el siglo siguiente- se presentan también en las discusiones de los principales exponentes de la cultura milanesa, en particular Giandomenico Romagnosi, Giuseppe Ferrari y Carlo Cattaneo, dispuestos a encontrar en Vico al primero en haber planteado el problema filosófico de la historicidad del actuar humano y del recorrido seguido por los pueblos en su camino hacia el "incivilimento". A partir de aquí, y del reforzamiento político y cultural de las relaciones entre la capital milanesa y la del reino meridional (sobre el que se construyó una pieza de la identidad de la nación), el recurso a la filosofía viquiana ha devenido imprescindible, identificando en el nexo entre lo verdadero y lo hecho, y entre lo verdadero y lo cierto, uno de los plexos problemáticos más importantes de la Scienza nuova, sobre el cual Vico reflexiona en particular a través de las famosísimas definiciones de una «teología civil de la providencia» y de una «historia ideal eterna». Definiciones, que parecen un oxímoron, que ponen de relieve la existencia de un carácter eterno y providencial, válido como medida de las acciones y conmensurado "por la debilidad del pensamiento humano», tanto como la relación entre el plano ideal y el fáctico, entre el nivel de los principios normativos y el de la capacidad creativa y psicológica del hombre, el cual conoce las cosas en su propia formación, constituye el punto a través del cual Vico desarrolla su propuesta de una ciencia de la historia.

Entonces, en las reflexiones de esos intérpretes de la primera mitad del Siglo Diecinueve, siempre informados del debate francés signado por las reflexiones de Victor Cousin, Eugène Lerminier, Pierre-Simon Ballanche, Theodor Simon Jouffroy y Jules Michelet, y, en algún caso (como es posible demostrar a través del estudio de la polémica entre Francesco Predari y Giuseppe Ferrari), anticipadores del largo 
debate sobre el "precursorismo", nace la conciencia de que el ritmo verdad-factualidad tiene una serie de consecuencias sobre la naturaleza "sociable" del hombre y sobre la historia de su civilización y permite razonar en torno a las "prácticas" de la Scienza nuova, desde el derecho hasta la historia, desde las lenguas hasta las etimologías, cuyos objetos e instrumentos están en relación con las estructuras ideales de la mente humana. No es posible aquí profundizar tales problemáticas de manera más específica. Las he recordado porque, a mi juicio, ellas han alimentado una parte importante de la cultura iluminista y democrática italiana, la cual se ha construido utilizando a Vico para indicar los caracteres de una filosofía práctico-civil conjugada con la reflexión sobre las diversas dimensiones del proceso de civilización humana. Desde este punto de vista son importantes las consideraciones de Giandomenico Romagnosi y su programa de fundación de una «filosofía civil», la cual, en cuanto «cognición de los principios directrices de la humanidad para lo mejor realmente obtenible por la vía de las causas coordinadas a la convivencia social», debe ser considerada una nueva forma de ciencia histórica. En una de sus obras más conocidas, Dell'indole e dei fattori dell 'incivilimento (1834), la «filosofía civil», de la cual uno se puede ocupar solo después de haber abandonado el campo de la «necesidad natural», es definida como la «ciencia de lo hecho en el proceso de civilización humana que abarca el perfeccionamiento económico, moral y político». Una ciencia, entonces, que debe ser capaz de mostrar cómo este proceso, desde el punto de vista humano y del desarrollo de los pueblos, es la cifra de un «dato complejo de funciones», de «factores» que, interactuando entre ellos, influyen sobre las condiciones psicológicas del hombre y sobre las condiciones civiles e históricas de las naciones, garantizando el progreso de la humanidad. Desde este punto de vista, rico en elementos viquianos, se muestra uno de los centros más importantes de la investigación romagnosiana, es decir, aquello que se refiere al nexo entre el hombre y el mundo de las naciones, construido para estudiar las funciones y las relaciones que se establecen entre las ideas y los hechos y capaz de destacar la acción "copotencial" que ellos realizan y hacen valer en la interacción entre el hombre y el mundo externo, los cuales actúan el uno sobre el otro modificándose recíprocamente. La filosofía civil, entonces, además de ocuparse del desarrollo de las naciones, se dirige al hombre y a sus modalidades cognoscitivas, en cuanto muestra cómo el hombre y la historia son el resultado de la combinación recíproca de factores internos y externos que, interactuando entre sí plenamente, construyen culturas fundacionales que actúan en el interior de la historia universal.

No obstante las reconocidas influencias ejercidas por el pensamiento romagnosiano, el itinerario "revolucionario" de Giuseppe Ferrari es distinto. Primer editor de las obras de Vico, es testigo de la idea compartida con Proudhon de que la filosofía no debe ser ya llamada para ayudar a los gobiernos, como quería el pensamiento de los reformistas meridionales, sino para ayudar a la revolución y a la jus- 
ticia. El estudioso milanés - cuya conocida monografía viquiana de 1837 recuerda, incluso por el título (La mente di Vico), la distinción fundamental entre la razón y la mente, la cual es solamente iluminada por las ideas- ofrece una de las primeras interpretaciones completas del itinerario viquiano, el cual encontraría su expresión más plena en la obra jurídica y en la Scienza nuova de 1725, a partir de la cual comenzaría una deriva de carácter metafísico. En el Discours sur l'histoire de la philosophie a l'èpoque de la Renaissance, editado en Estrasburgo en 1840, Ferrari, después de haber indicado que en el Renacimiento comienza el mundo moderno, inicia una serie de consideraciones en torno a una «ciencia nueva», es decir, la historia de la filosofía, válida para interrogarse sobre los modos para estudiarla y sobre la utilidad que ella puede conseguir. Aquí el discurso en torno a la historia es afrontado desde dos puntos de vista distintos: el interno, que considera el desarrollo de las ideas y del espíritu humano (que es "prédéterminée par les lois qui le dirigent») - de tal manera que la historia de la humanidad se presenta como una continuación de la psicología-, y el externo, donde todos los acontecimientos deben ser considerados accidentales. Sobre la base de esta premisa, la historia de la filosofía es acercada a la historia de la sociedad y ambas son consideradas paralelamente y como capaces de influir la una en la otra (aun con la conciencia de que el encuentro entre el mundo intelectual y el mundo físico queda siempre como un «mystère»). La nueva ciencia de la historia (de la cual la historia de la filosofía ocupa solo una parte) tiene su ubicación en el mundo de las ideas y del espíritu, y si los elementos necesarios en la historia son o bien los principios o bien las consecuencias que se derivan de ellos, entre estos dos extremos se despliegan millares de sistemas, que representan los lugares en torno a los cuales las ideas, los principios, se agregan permitiendo progresar o retroceder a la humanidad. Entonces esto delinea dos planos de la «ciencia de la historia»: el que «corresponde a la historia positiva» y el que interesa a «la ciencia de la historia de la filosofía», de la sucesión abstracta de las ideas y de los principios que constituyen los dos niveles en torno a los cuales se articula el discurso ferrariano sobre la nueva ciencia de la historia, la que encuentra su desembocadura fundante en el Essai sur le principe et les limites de la Philosophie de l'histoire, editado en París en 1845.

En esta obra compleja es puesta de manifiesto la consideración según la cual, en la historia, los hechos son subordinados al acaso y a la fatalidad, de modo que para juzgarlos, para interpretarlos, para relatarlos es necesario un principio capaz de mantener unidos el método a priori y el a posteriori, clasificando los hechos según las leyes del intelecto a través del estudio de los hechos. En efecto, solo en el campo ideal pueden ser satisfechas las condiciones del método a priori y las del método a posteriori, ya que en un sistema ideal hay necesidad tanto de los hechos positivos, que el método a priori exige para salir de su indeterminación, cuanto de los hechos racionales, es decir, de las "anticipaciones" supuestas por el 
método a posteriori que evitan que los hechos mismos tengan todos la misma importancia. La nueva ciencia de la filosofía de la historia se transforma en una filosofía de lo ideal, una demostración abstracta de los progresos de la razón, que debe encontrar su punto de llegada en un sistema donde, en la brecha entre la naturaleza y lo ideal es posible pensar una ciencia absoluta capaz de justificar la utopía y el misticismo. Se puede entonces afirmar que, haciendo propia de modo original la lección viquiana, el plano teórico se comprueba en el histórico-efectual para determinar el desarrollo general de la humanidad, legitimando la tendencia futura, así como la Teoria dell'umanità, la cual, después del análisis de las ideas y de la inteligencia del hombre y del dedicado a la historia ideal, concluye el Essai, que tiene la misión de conjugar el curso de las naciones con el ideal de la humanidad, superando todas las contraposiciones entre la historia ideal eterna y la historia positiva. Los resultados alcanzados, en particular los de la tercera parte, son luego profundizados en la Filosofia della rivoluzione (1851), la obra en la cual, partiendo del rechazo de todo enfoque dogmático, de todo sistema que quiera ponerse como absoluto, la filosofía, siendo el elemento característico de la condición humana, de la razón común a todos los hombres, puede convertirse en «revolución». Desde esta perspectiva, ella se centra en dos principios fundamentales, la verdad y la justicia, los cuales, aunque subordinados al riesgo de las contradicciones lógicas de las concepciones tradicionales, son capaces de atacar todas las estructuras del poder, las oligarquías y los aparatos burocráticos y permitir a la verdad conquistar el «hecho» y de dar inicio, junto con la justicia, al reino de la ciencia y de la igualdad. Es necesario, entonces, suspender el juicio sobre los conocimientos formales y abstractos y hacer emerger lo que es evidente, vale decir el hecho, lo único capaz de mostrar que las cosas están frente a la conciencia como realmente son en su verdad-multiplicidad, en su continuo devenir. Reconquistado el «hecho», y rechazada toda amenaza lógica o metafísica, Ferrari declara haber realizado la inversión de la perspectiva filosófica tradicional: mientras el pensamiento que nos revela las contradicciones del mundo parece forzarnos a abandonarlo para buscar una realidad sólida en otra parte, la filosofía de la revolución, que es una verdadera filosofía liberadora, revela que las contradicciones y los errores muestran solo la insuficiencia del pensamiento frente a la vida. La Filosofia della rivoluzione, entonces, quiere retornar a los acontecimientos del mundo rechazando la lógica y abriéndose a la revelación, a la «revelación natural», que se manifiesta primero en la «revelación de los seres»o de las cosas, luego en la «revelación de la vida»y, por último, en la «revelación moral», haciendo que la vida se imponga con su imperativo: es necesario que se viva apropiándose de todas las modalidades posibles, las materiales, las instintivas, las morales y las sociales. En su conjunto, la filosofía de la historia ferrariana -que delinea también un desarrollo progresivo en los tres estadios de la religión, de la metafísica y de la ciencia (estadio en el que la revolución se realiza a través de sus 
principios fundamentales de la ciencia y de la igualdad), atacando tanto las ideologías providencialistas-escatológicas, como las materialistas, y sobre la base de la interpretación fundamental y original de la reflexión viquiana- construye una concepción de la historia fundada sobre el progreso y sobre el desarrollo de las ideas y, por tanto, caracterizada por una tensión continua hacia la libertad y hacia la justicia vivida en la conciencia del riesgo del error y de la caída. A partir de una concepción tal, Ferrari, aun indicando el progreso y la regresión de los sistemas sociales, apunta a la idea de una «asociación universal», que es el principio de la humanidad implícito en todo hombre y en toda nación. En el interior de este cuadro, Vico constituye al fin y al cabo un momento, aunque esencial, de una teoría de la historia ideal eterna, ya que si para el filósofo de la Scienza nuova el elemento determinante está constituido por la nación, para Ferrari la evolución de los pueblos hacia la civilización es un momento de una historia que conoce también una fase ulterior, en la cual es rastreable una forma "utópica" marcada por una exigencia de libertad y de justicia que, obstaculizada por esos sistemas que le bloquean el camino, le permiten elaborar un proyecto político de tipo federativo aplicado a la realidad de la contingencia histórica. Sin embargo, también aquí continúan actuando elementos viquianos en cuanto la historia es entendida como un lugar de contrastes, de conflictos de fuerza, donde lo útil deviene la ocasión para relacionarse con el mundo externo y donde el acento cae sobre la modalidad del hacer humano, conectada a una idea de la realidad como mutación continua, que aleja lo verdadero en el sentido de la pretensión absoluta de entenderlo en la práctica de la subjetividad humana. De ello deriva la actitud que, rechazando críticamente el estado de cosas existentes, se propone favorecer una concepción política y social dinámica, en continua transformación, por la cual, en Ferrari, la historia ideal debe llegar a ser historia social y la socialidad es entendida como el nexo del progresivo proceso de civilización humana, con el que se afirman la ciencia y la justicia, esto es: las verdaderas armas de la filosofía de la revolución. En ese sentido, si Vico le reconoce al orden providencial la tarea de ejecutar un orden en la historia, sujeto precisamente a la norma ideal de la providencia, quizás se puede decir que Ferrari identifica en la providencia o la fatalidad («el principio que rige sobre los pensamientos de los hombres y sobre las cosas de este mundo, la Diosa de todas las revoluciones republicanas y dinásticas»), la respuesta a las contradicciones de la realidad, recuperando la praxis y la voluntad humana como el fundamento de la historia, donde es la socialidad la que promueve el camino. Sin embargo, también en este ámbito la dimensión ideal no va separada de la consideración empírica, que en la Filosofia della rivoluzione permite un estudio del hombre en su fisicalidad primaria, es decir, la esfera donde el significado histórico social contiene dentro de sí un "antes" originario. Es interesante reconocer que tal programa filosófico no es separable del programa político de Ferrari, como demuestran sobre todo las páginas de La federazione repubblicana, editado el mismo año 
en que publicaba la Filosofia della rivoluzione, en las cuales es indicado el camino a través del cual liberarse de los antiguos errores, del dominio temporal y espiritual de güelfos y gibelinos, que figuran entre las causas de la «esclavitud italiana», y buscar las «vías de la liberación». En el estudioso milanés, impregnado profundamente por los estudios y las problemáticas viquianas, el proyecto político de emancipación de Italia, que debía pasar a través del sacrificio del poder imperial y del poder papal, habría debido guiar el proceso de unificación nacional no según los principios "formalistas" del mazzinismo, sino gracias a una «revolución social» que, conectada con la revolución de los filósofos, debía ser capaz de fundar el socialismo contra el papado y el imperio, el modo de luchar contra los hechos con las ideas, contra «un orden material, antiguo, arraigado en las leyes, en los intereses, en las creencias» con un «orden invisible», aún por construir. En este cuadro son muchos los elementos que Ferrari discute libremente con algunos autores de la tradición filosófica francesa, a partir de Comte, cuya teoría subyace claramente al triple movimiento de la revelación, pero también Pierre Leroux y Proudhon, el cual, además de interrogarse sobre la idea de asociación como libre iniciativa de los trabajadores y de los grupos, había trabajado sobre el principio de autoridad, sosteniendo que la religión así como la moral, la justicia, las leyes, no se deben a una revelación externa que se impone a los hombres desde lo alto, sino que pertenece a la naturaleza humana y sigue la evolución de la sociedad.

Respecto al cuadro aquí esbozado, las ideas viquianas aparecen orientadas de modo diverso, que asimismo continúan siendo presentadas en la posición de un muy conocido estudioso lombardo de aquella época, Carlo Cattaneo, quien, rechazada la idea de reducir la filosofía a historia de la filosofía, se dirige a una «revolución de la filosofía» practicada a través de la reflexión sobre la naturaleza (Bacon), sobre el individuo (Locke) y sobre la sociedad (Vico). Una reflexión que parte de la distinción entre individuo y sociedad, articulada gracias a la diferenciación ente una psicología del individuo y una ideología de la sociedad (que es el estudio del individuo en el seno de la humanidad). Si la psicología es el estudio de las facultades del alma, y la ideología el estudio de la formación de nuestras ideas por esas facultades, psicología e ideología son unidas por la exigencia de ofrecer una dirección "social" a sus investigaciones, y mientras una considera al agente, la otra se dirige a las obras y las ideas, las cuales, respecto al agente, que es siempre el mismo, varían continuamente, avanzando y retrocediendo. Así, dado que la psicología y la ideología del individuo abstracto no logran dar cuenta de la variedad de las ideas y de las costumbres, se vuelven necesarias una psicología y una ideología sociales capaces de explicar la historia. La psicología social, partiendo de la afirmación de que el acto más social del hombre es el pensamiento, tiende a mostrar que tanto las facultades (la sensación, la memoria y la imaginación), como las operaciones mentales (v.g. el análisis), y como las más altas creaciones del pensamiento (los sistemas ideales y 
las ciencias) no se pueden explicar más allá de la sociedad, en la que las primeras se desarrollan, las segundas se ejercitan y las últimas se producen. La psicología social, por tanto, es el presupuesto necesario de la ideología social, así que solo mostrando que las facultades y las operaciones de la mente se modifican por obra de las mutuas relaciones entre los hombres se crean las bases para comprender cómo se forman y transforman las ideas. Los presupuestos de los que la ideología social deriva la propia legitimidad son dos: por un lado, la historia de la civilización en cuanto historia de las ideas (son las ideas, en efecto, lo que hacen ser a una civilización lo que ella es), en la que la inteligencia, que es la facultad indagadora y ordenadora, pero también inventora, debe entenderse como la gran potencia creadora del mundo humano y de su historia; por el otro, el hecho de que las ideas son un producto de la vida en sociedad, y que solo el estudio de la historia de las sociedades humanas, en los más diversos tiempos y latitudes, puede ofrecer los documentos necesarios a la nueva ciencia, la cual procura ser una filosofía experimental de la historia, pero también el campo de la «libertad moral y de la responsable potencia del individuo». Con Cattaneo el itinerario viquiano encuentra un resultado original en el descubrimiento de la centralidad de la individualidad, de la conciencia individual, indivisible de la moralidad, de los pueblos singulares, de las historias singulares, que luego, mediante sus interacciones mutuas, devienen la historia de la humanidad, historia universal. La filosofía civil cattaneana se mueve entonces hacia una dirección distinta a la ferrariana. En efecto, rechazando la idea de que «toda civilización forma necesariamente un sistema, el cual no puede jamás caer si no es por la sustitución de otro sistema» (lo que significa rechazar la concepción según la cual, siendo la historia una sucesión de sistemas, la sociedad es un sistema que no tolera contradicciones, y cuando estas se desarrollan, ella está destinada a morir, haciéndose sustituir por otro sistema), Cattaneo ataca el punto crucial de la perspectiva ferrariana, fundada, podemos decir rosminianamente, sobre una idea primera, absoluta, de la cual extraen su origen todas las otras ideas. Él, en cambio, sostiene que la multiplicidad de los principios, con su consiguiente conflictividad y contradictoriedad, permite el progreso de las naciones y de las civilizaciones, de modo que «cuanto más civil es un pueblo, tanto más numerosos son los principios que contiene en su seno». La historia, por tanto, «es el eterno contraste entre diversos principios que tienden a absorber y a uniformar las naciones», de modo que ella no es jamás inmóvil, ni expresable en improbables proyectos de paz perpetua o de repúblicas universales, ni es la formación de sistemas de ideas que se suceden el uno al otro, sino más bien una construcción de órdenes civiles, en los cuales la serie de los datos, operando sobre el intelecto, producen los «cambios de escena», es decir, nuevos sistemas en los que aparecen principios destinados a renovarse y transformarse. Por esto, solo la adopción de nuevas formas de ideas y el pasaje a una fase ulterior de la civilización puede cambiar los equilibrios obtenidos, por los que los inte- 
reses y los impulsos de la voluntad son orientados siempre hacia una más amplia perspectiva económica, política y cultural y hacia la libertad moral, mediante la cual se pueden realizar las distintas transiciones. La comparación con Ferrari revela, precisamente, que las reflexiones cattaneanas son alimentadas por la idea de que a los "sistemas" cerrados se deben contraponer los abiertos, de modo que se puede sostener que solo las sociedades en movimiento y en transformación, diferenciándose de las "estacionarias", se modifican no sobre la base de elementos "revolucionarios", sino gradualmente, de modo que en ellas no se asiste al derrocamiento de un sistema por otro, sino más bien al «traspaso» lento y nebuloso «de la animalidad inconsciente a la bella y sublime racionalidad», que implica justamente la validez de la gradualidad del proceso histórico por todas las épocas.

[Traducción del italiano por Alberto Mario Damiani]

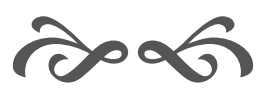

